\title{
Presencia de mercurio en cuenca del rio Napo
}

\author{
Duma Luz Rengifo Pinedo ${ }^{1}$, Wilson Reyes Lazaro², Lindsay Prado Torres ${ }^{1}$ \\ ${ }^{1}$ Universidad Nacional de la amazonia peruana, (UNAP) Iquitos - Perú. \\ ${ }^{2}$ Universidad Nacional de Trujillo, (UNT) Trujillo - Perú.
}

\begin{abstract}
Resumen
La investigación se enfoca en la problemática ambiental generada por el manejo inadecuado de los materiales y residuos peligrosos proveniente de actividades mineras importantes en la amazonia, especialmente la aurífera informal, realizada en forma artesanal en la zona del bajo Napo [1]. Nuestro propósito es identificar presencia de mercurio en la población a partir del consumo de pescado y su confirmación en el río Napo, también colaborar en la prevención para mejorar la calidad de vida humana y a la vez sensibilizar sobre la necesidad de una vigilancia permanente del ambiente para resguardar las condiciones de vida de los pobladores de Santa Clotilde - Mazan y de otras zonas expuestas. La investigación es aplicada - descriptiva, de diseño no experimental y transversal [2]. El área de estudio es el rio Napo ubicada en la región Loreto, comprende cuatro estaciones: (Mazan, Nuevo Libertad, San Luis, Santa Clotilde). Se consideró evaluar aguas, y peces del río Napo. La cuantificación de mercurio se realizó mediante espectrofotometría de absorción atómica con generador de hidruros [3] Técnica Oficial: AOAC N 977.15 [4]. Los análisis reportan que el contenido de mercurio en peces se encuentra entre $0.004-1.936 \mathrm{mg} / \mathrm{kg}$, siendo su valor promedio $0.607 \mathrm{mg} / \mathrm{kg}$ y en aguas se encuentra entre $0.023-0.027 \mathrm{mg} / \mathrm{L}$ siendo su valor promedio $0.024 \mathrm{mg} / \mathrm{L}$. Sin embargo en las muestras de peces de las especies Lisa y Sábalo, pasaron por encima de la concentración máxima permitida $(1.0 \mathrm{mg} / \mathrm{kg}$ de mercurio) establecido por la FDA (Food and Drug Administration) de los Estados Unidos de América [5]. El promedio de consumo mensual de pescado en el ámbito local se encuentra entre $15 \mathrm{a} 20 \mathrm{Kg}$ de pescado por mes, obteniendo 10,622.5 $\mathrm{\mu g} \mathrm{Hg}$ mensualmente y $127,470 \mu \mathrm{g} \mathrm{Hg}$ al Año de acuerdo a los resultados. La retención de Mercurio por el consumo de pescado está por determinarse, sin embargo por consumo de Mercurio líquido la retención es del $0.01 \%$ en el tracto gastrointestinal [6]. Si en el tracto gastrointestinal se retiene el $0.01 \%$ del consumo de pescado, entonces de acuerdo a lo obtenido $12.747 \mu \mathrm{Hg}$ quedara retenida anualmente en el organismo, lo que excede a lo permisible por la Organización Mundial de la Salud (OMS) de $0.0014 \mu \mathrm{g}$ de $\mathrm{Hg}$ anual. Además el contenido de mercurio encontrado en agua supera a lo establecido en los Estándares Nacionales-MINAM (0.0001 mg/L de $\mathrm{Hg}$ ). Inclusive superando al reporte de la DIGESA, en Informe $\mathrm{N}^{\circ} 3527-06,3527-07,3527-12(2003)$ y N 3935 (2010), cuyo reporte es $0.00002 \mathrm{mg} / \mathrm{L}$ de $\mathrm{Hg}$, valores obtenidos de los puntos de muestreos de Santa Clotilde, San Luis y Mazan, en la cuenca del rio Napo [7]. Finalmente, concluimos que existe presencia importante de mercurio en agua y peces del río Napo. Así también confirmamos que las concentraciones de mercurio en las estaciones monitoreadas, son mayores a los Estándares Nacionales de Calidad Ambiental, por lo que urge una evaluación de mayor amplitud, teniendo en cuenta otros bioindicadores (cabello, sangre, orina), la que nos confirmara la presencia de este elemento toxico en la cadena alimenticia y en el ecosistema, es más nos brinda la oportunidad de proponer y ejecutar estrategias de vigilancia en nuestros ríos amazónicos en riesgo por el desarrollo de la actividad minera informal.
\end{abstract}

Descriptores: Mercurio, Sábalos, Toxicidad, Calidad ambiental, Rio Napo. 


\begin{abstract}
The research focuses on environmental problems caused by improper handling of hazardous materials and wastes from major mining activities in the Amazon, especially the informal gold held in a small scale in the area of the lower Napo [1]. Our purpose is to identify the presence of mercury in the stock from fish consumption and its confirmation in the Napo River, we are also working on prevention to improve the quality of human life while raising awareness of the need for ongoing monitoring of the environment to improve and protect the lives of the residents of Santa Clotilde - Mazan and other exposed areas. The research is applied descriptive, cross-sectional unexperimental design [2]. The study area is located in the Napo region of Loreto, has four seasons: (Mazan, Nuevo Libertad, San Luis, Santa Clotilde). Was considered to evaluate water and fish,from the river Napo. The quantification of mercury was performed by atomic absorption spectrophotometry with hydride generator [3] Technical Officer: No. AOAC 977.15 [4]. Analysis report that the mercury content in fish is between $0.004-1.936 \mathrm{mg} / \mathrm{kg}$, and its average value and $0.607 \mathrm{mg} / \mathrm{kg}$ water is between $0.023-0.027 \mathrm{mg} / \mathrm{l}$, average value being $0.024 \mathrm{mg} / \mathrm{l}$. However, in samples of fish species Lisa and Sabalo, went above the maximum allowable concentration $(1.0 \mathrm{mg} / \mathrm{kg}$ of mercury) established by the FDA (Food and Drug Administration) of the United States of America [5]. The average monthly consumption of fish at the local level is between 15 to $20 \mathrm{~kg}$ of fish per month. Obtaining 10,622.5 $\mu \mathrm{g} \mathrm{Hg} / \mathrm{g}$ monthly and 127,470 $\mathrm{mg} \mathrm{Hg} / \mathrm{g}$, year according to the results. The retention of mercury by eating fish is to be determined; however the of Liquid mercury retention from consumption is $0.01 \%$ in gastrointestinal tract [6]. If in the gastrointestinal tract is held $0.01 \%$ of fish consumption, then according to that obtained $12,747 \mu \mathrm{g} \mathrm{Hg}$ will be retained in the body annually, which exceeds those allowed by the World Health Organization (WHO) of $0.0014 \mu \mathrm{g}$ of $\mathrm{Hg}$ annual, and the content of mercury found in water exceeds the provisions of the National Standards-MINAM $(0.0001 \mathrm{mg} / \mathrm{L}$ of $\mathrm{Hg}$ ), even beating the report of the DIGESA. In Report No. 3527-06, 3527-07, 3527-12 (2003) and No. 3935 (2010), whose report is $0.00002 \mathrm{mg} / \mathrm{L}$ of $\mathrm{Hg}$, values obtained from sampling points of Santa Clotilde, San Luis and Mazan, in the Napo River basin [7]. Finally, we conclude that there is significant presence of mercury in water and fish from the river Napo. This also confirmed that mercury concentrations in the monitored stations, are higher than the National Environmental Quality Standards and it urges a broader assessment, taking into account other bio-indicators (hair, blood, urine), we confirm the presence of this toxic element in the food chain and ecosystem, it is gives us the opportunity to design, propose and implement monitoring strategies in our Amazon rivers at risk for the development of informal mining activities.
\end{abstract}

Keywords: Mercury, Sábalos, toxicity, environmental quality, Rio Napo.

\section{Introducción}

Los metales pesados son considerados los contaminantes más problemáticos de los ecosistemas acuáticos, siendo una de las razones, su capacidad para formar complejos con la materia orgánica presente tanto en el agua como en los sedimentos y por la tendencia de estos elementos metálicos de fijarse en los tejidos de los organismos expuestos $^{8}$. Los metales pesados en aguas naturales pueden existir en la forma de iones libres, unidos a carbonatos solubles, sulfuros, hidróxidos, cloruros o sulfatos y como complejos solubles con ligandos Orgánicos ${ }^{9}$.
La contaminación del agua puede ser de múltiples formas, según la procedencia de los desechos; por sedimentos, materia orgánica, biocidas, metales pesados y otros elementos tóxicos que provocan distintos grados de impactos sobre las características físico-químicas propias del agua, sobre la flora, la fauna y el hombre. Investigaciones reportan que en la extracción aurífera, la mediana minería arroja 3 toneladas de mercurio anual; la pequeña minería arroja otro tanto, en total 6 toneladas anuales, equivalente a 24 kilos de mercurio por kilómetro cuadrado de río, esta es la cantidad de mercurio que se introduce a las aguas amazónicas cada año, durante el proceso de separación, el mercurio se evapora y estos vapores 
generan una contaminación directa en los trabajadores; otra parte es depositada en las aguas, ingresando así en la cadena trófica y llegando a la población que los consume ${ }^{1}$, reportes existentes indican que el mercurio está presente especialmente en peces, en concentraciones perjudiciales para los seres humanos y en la flora y fauna silvestres. Tales niveles han ocasionado que en algunos países se formulen recomendaciones sobre el consumo de pescado, sobre todo los subgrupos vulnerables (como mujeres embarazadas y niños) reduzca o evite el consumo de ciertos tipos de pescado provenientes de distintas aguas. No es probable que el consumo moderado de pescado (con niveles bajos de mercurio) ocasione exposiciones de consideración. En cambio, la población que consume grandes cantidades de pescados contaminados puede quedar muy expuesta al mercurio $y$, por consiguiente, se encuentra en riesgo. ${ }^{10}$ Por tal motivo es importante conocer el contenido de mercurio en peces, sedimentos y aguas, objetivo de este trabajo para obtener un diagnóstico del ecosistema materia importante de este estudio, propósito que nos llevara a mejorar las condiciones de vida de esta zona Amazónica (Mazan, Nuevo Libertad, San Luis y Santa Clotilde.)

\section{Metodología}

\section{Descripción del área de Estudio:}

El área de estudio es el rio Napo ubicada en la región Loreto, comprende cuatro (4) estaciones: Mazan, Nuevo Libertad, San Luis, Santa Clotilde.) Ver Fig. 1

Especímenes de estudio: Se consideró la evaluación de aguas y peces del río Napo. Especímenes: bagres (Pimelodella sp), sábalo (Brycon americus) y lisa (calossomani gripinis),

La recolección de muestras de aguas y peces fue realizada considerando previo tratamiento de los materiales, libres de impurezas que puedan hacer una reacción con el metal inorgánico.

1 CIED, Medio Ambiente. 1993.

8 Ávila P. Evaluación de los metales pesados 1995.

${ }^{9}$ Chapman D. Water quality assessments. 1992.

${ }^{10}$ Evaluación Mundial sobre el Mercurio. 2002.

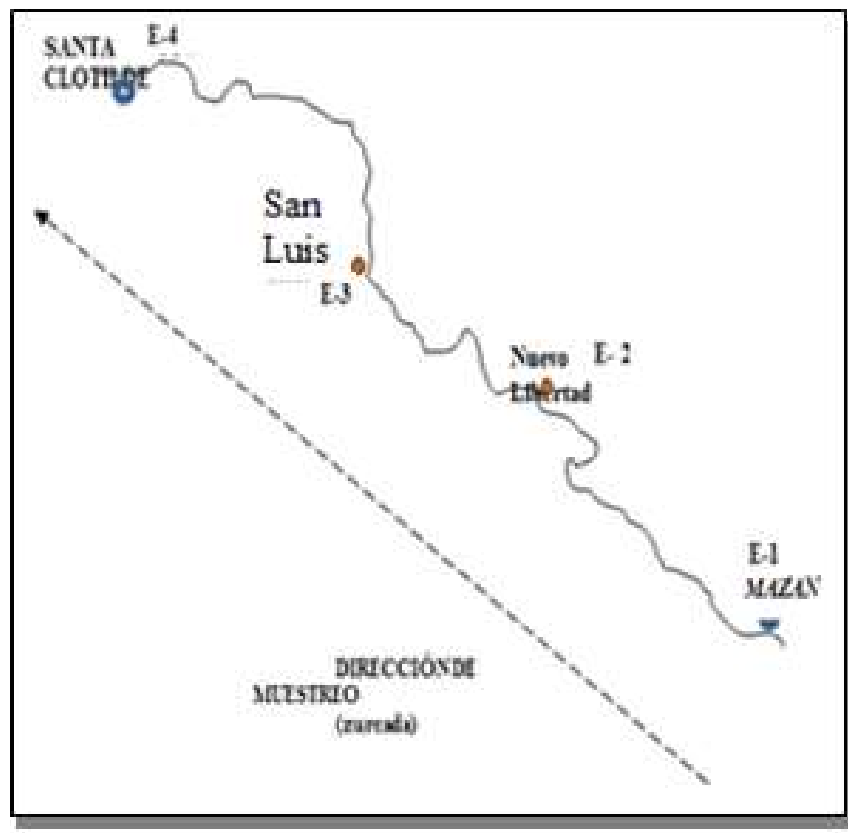

Fig.1: Monitoreo Mazan-Santa Clotilde, río Napo

Aguas: se tomó muestras de puntos representativos de la masa liquida. (a superficie, $30 \mathrm{~cm}$ bajo superficie, $100 \mathrm{~cm}$ bajo superficie) de las tres partes de la zona. (margen izquierdo, medio, margen derecho) del cuerpo principal. La toma de muestra se realizó en envases previamente acondicionados para el propósito, limpios, libres de impurezas.

Peces. Los especímenes colectados durante el monitoreo fueron: bagres (Pimelodella sp), sábalo (Brycon americus) y lisa (calossomani gripinis), los que fueron seleccionados, identificados, medidos y pesados para luego colocarlos en bolsas plásticas y conservarlos en hielo para su transporte al laboratorio. Las muestras para el análisis, fueron obtenidas empleando cuchillos plásticos a partir del músculo dorsal cortando aproximadamente de 5 a 20 gramos de cada espécimen, conservados en frascos plásticos a una temperatura de $-20^{\circ} \mathrm{C}$ hasta su análisis. Los especímenes (peces) fueron analizados para la determinación de concentración de mercurio en tejido, colectándose 14 individuos: bagres (2), lisa (6) y sábalos (6) por cada área de estudio de acuerdo a la zona de evaluación, (Fig.1) 
Análisis químico y cuantificación de $\mathrm{Hg}$ en muestras.

Digestión ácida, durante este proceso las muestras son solubilizadas y el mercurio es liberado de la matriz biológica o ambiental. ${ }^{5}$

En base a método de la AOAC. ${ }^{4}$ Método 977.15. En este proceso de digestión todas las muestras analizadas fueron digeridas utilizando una mezcla de ácidos nítrico y sulfúrico.

Para la digestión y cuantificación de Hg en peces, se pesó $1.5 \mathrm{~g}$ de pescado al cual se agregó $5 \mathrm{ml}$ de agua desionizada y $5 \mathrm{ml}$ de $\mathrm{H}_{2} \mathrm{SO}_{4}$, durante el calentamiento se adiciono gota a gota $2 \mathrm{ml}$ de Peróxido de Hidrogeno al $30 \%$, luego se dejó en reposo la mezcla de ácidos a temperatura ambiente durante $12 \mathrm{~h}$. Luego terminado el proceso, se filtró y se preparó la dilución: 150ul de la solución acida en $10 \mathrm{ml}$ de agua desionizada, ${ }^{4,5}$ para luego proceder a su cuantificación mediante el análisis por instrumentación (EAA-generador de hidruros).

\section{Digestión de Aguas}

Para la conservación del Análito $(\mathrm{Hg})$ se adiciono HNO3 y permaneció en refrigeración hasta su análisis. Luego se procedió a preparar la dilución: $200 u$ de la solución acida en $10 \mathrm{ml}$ de agua desionizada, para luego proceder a su cuantificación mediante el análisis por instrumentación (EAAgenerador de hidruros).

La cuantificación de $\mathrm{Hg}$ de todas las muestras (peces y aguas) se realizó por medio de Espectrofotometría de Absorción Atómica generadorhidruros en base a Magos y Clarkson (1972). Efectuando un control de calidad ${ }^{3}$ y el Standard de $\mathrm{Hg}$ fue usado como material de referencia.

\section{Resultados y discusión}

Los análisis reportan que el contenido de mercurio en peces se encuentra entre $0.004-1.936 \mathrm{mg} / \mathrm{kg}$, siendo su valor promedio $0.607 \mathrm{mg} / \mathrm{kg}$ y en aguas se encuentra entre $0.023-0.027 \mathrm{mg} / \mathrm{l}$ siendo su valor promedio $0.024 \mathrm{mg} / \mathrm{l}$. Sin embargo en las muestras de peces de las especies Lisa y Sábalo, pasaron por encima de la concentración máxima permitida (1.0 $\mathrm{mg} / \mathrm{kg}$ de mercurio) establecido por la FDA (Food and Drug Administration) de los Estados Unidos de América $^{3}$. Ver figura 02.

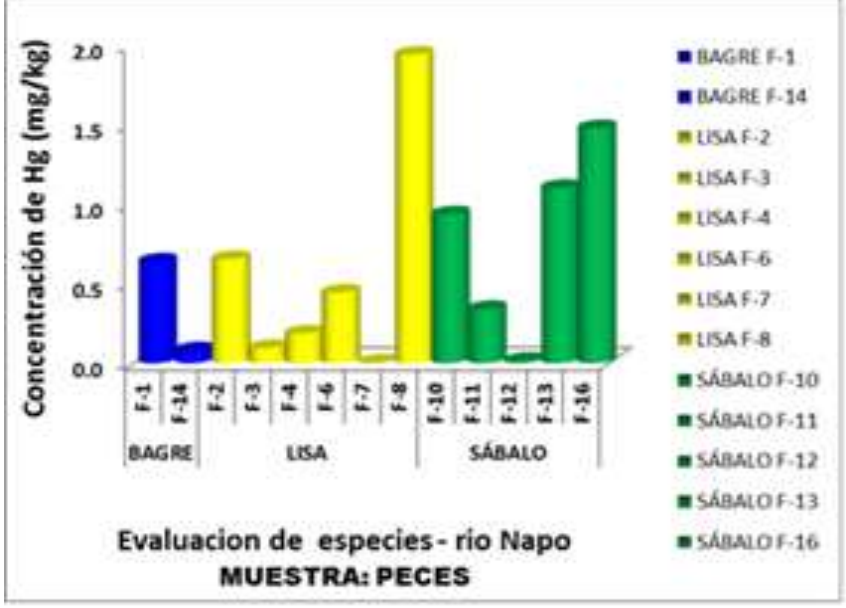

Fig. 2: Evaluación de Mercurio en peces

En la Figura 2, se observa que algunas especies lisa (F8) y sábalos (F13, F16), sobrepasaron el limite permisible de concentración máxima de $1.0 \mathrm{mg} / \mathrm{Kg}$ de mercurio.

El promedio de consumo mensual de pescado en el ámbito local se encuentra entre 15 a $20 \mathrm{Kg}$ de pescado por mes. $17,500 \mathrm{~g} \times 0.607 \mu \mathrm{Hg} / \mathrm{g}=$ $10,622.5 \mu \mathrm{g} \mathrm{Hg}$ mensualmente y $127,470 \mu \mathrm{g} \mathrm{Hg}$ al Año. La retención de Mercurio por el consumo de pescado está por determinarse, sin embargo por consumo de Mercurio líquido la retención es del $0.01 \%$ en el tracto gastrointestinal ${ }^{6}$. Si en el tracto gastrointestinal se retiene el $0.01 \%$ del consumo de pescado tenemos que: $127,470 \mu \mathrm{Hg}$ anual $\mathrm{x}$ $0.01 \%=12.747 \mu \mathrm{g} \mathrm{Hg}$ retenidas anualmente lo que excede a lo permisible por la Organización Mundial de la Salud (OMS) de $0.0014 \mu \mathrm{g}$ de $\mathrm{Hg}$ anual.

Entre los resultados del contenido de $\mathrm{Hg}$ en las muestras de aguas de las diferentes estaciones mínimamente oscilan entre el rango de concentración como sigue: para Mazan entre 0.023 - $0.025 \mathrm{mg} / \mathrm{l}$ de $\mathrm{Hg}$, Nuevo Libertad entre 0.023 0.026, mg/l de Hg, San Luis entre 0.022-0.025 mg/l de $\mathrm{Hg}$ y para Santa Clotilde en el rango de 0.023 $0.027 \mathrm{mg} / \mathrm{l}$ de $\mathrm{Hg}$ Ver. tabla 01.

3 Magos y Clarkson, Atomic absorption 1972.

4 AOAC Official Method 977.15 Mercury in Fish

5 Sadiq, Sample weight and digestion 1991.

6 Donna Megler, Contaminación en la Cuenca del rio nanay 
Tabla 1: Concentración de $\mathrm{Hg} \mathrm{mg} / \mathrm{l}$. en aguas zonas estratificadas. Margen derecho, Medio e Izquierdo y Niveles de profundidad en las áreas de estudio

\begin{tabular}{|c|c|c|c|c|c|c|}
\hline Ubicación & \multicolumn{2}{|c|}{ Margen Derecho (2m) } & \multicolumn{2}{|c|}{ Medio } & \multicolumn{2}{c|}{ Margen Izquierdo (2m) } \\
\hline Area & $70 \mathrm{~cm}$ & $30 \mathrm{~cm}$ & $30 \mathrm{~cm}$ & $100 \mathrm{~cm}$ & $30 \mathrm{~cm}$ & $70 \mathrm{~cm}$ \\
\hline Mazan & 0.025 & 0.023 & 0.024 & 0.024 & 0.023 & 0.025 \\
\hline Nvo Libertad & 0.026 & 0.025 & 0.024 & 0.024 & 0.023 & 0.023 \\
\hline San Luis & 0.023 & 0.024 & $*$ & 0.022 & 0.025 & 0.024 \\
\hline Sta Clotilde & 0.023 & 0.024 & $*$ & 0.023 & 0.025 & 0.027 \\
\hline
\end{tabular}

* Ninguna muestra

En las muestras de aguas analizadas de las estaciones a diferentes niveles de profundidad el contenido de mercurio encontrado en agua de estas zonas superan a lo establecido en los Estándares Nacionales de Calidad Ambiental ${ }^{11} \mathrm{~N}^{\circ}$ 002-2008MINAM (0.0001 mg/L de Hg), para los cuerpos de agua catalogados en la categoría 4: Conservación del Ambiente Acuático (Selva). Aguas de preservación de fauna acuática y pesca recreativa o comercial. Inclusive superando al reporte de la DIGESA, en Informe $\mathrm{N}^{\circ} 3527-06,3527-07,3527-$ 12(2003) y No 3935 (2010), cuyo reporte es 0.00002 $\mathrm{mg} / \mathrm{L}$ de $\mathrm{Hg}$, valores obtenidos de los puntos de muestreos de Santa Clotilde, San Luis y Mazan, en la cuenca del rio $\mathrm{Napo}^{7}$.

\section{Conclusiones}

El mercurio se encuentra actualmente en diversos estratos y componentes biológicos del tramo estudiado (Santa Clotilde - Mazan) niveles que afectan adversamente a la vida silvestre. La actividad cotidiana del hombre ha generalizado los casos de exposición a quien afecta indudablemente. Las prácticas del pasado han dejado un legado de mercurio como depósito en nuestros ríos, los que algún día podrían ser liberados posiblemente por efecto de la remoción de nuestra vegetación y corteza terrestre.

Los desechos de la minería y los emplazamientos, provocaron que aguas y peces se encuentren contaminados de mercurio en la zona del Rio Napo, ocasionado por la presencia de altas concentraciones de mercurio inorgánico encontradas en el agua y ligeramente elevados en peces. En el agua, el mercurio biodisponible supera muchas veces el nivel máximo permitido para la protección de la vida acuática.

Se encontró presencia de mercurio en aguas, valores que exceden el Límite Máximo Permisible (LMP) para su uso como fuente de abastecimiento de agua potable y para la protección de la vida acuática, lo que indica que es necesario vigilar, controlar y proteger de forma continua el cumplimiento de las Normas Legales que nos aseguran el derecho de vivir en un ambiente natural y protegido de cualquier riesgo de toxicidad ocasionado por la presencia de mercurio u otra sustancia que dañe a nuestra amazonia

\section{Agradecimientos}

Agradecimiento al Dr. Noé Costilla por brindarme las facilidades en la Universidad Nacional de Trujillo UNT; para la ejecución de los análisis.

Dr. Wilson Reyes L. por su valioso aporte a la ciencia y aceptar ser mi mentor en esta etapa Profesional, al Ing. Roger Beuseville-IIAP, por el soporte técnico prestado, Ing. León Bendayan-IIAP por el apoyo prestado, Ing. José Sanjurjo-IIAP por la afinación técnica de la georeferenciación de los puntos de muestreo, a mis profesores, sabios maestros en la conducción de nuestro aprendizaje.

\section{Referencias}

[1] CIED, Medio ambiente. Set, Lima, 56 (1993) 45

[2] H. S. Roberto, Metodología de la Investigación. 4ta.Edición. Edit. Mc Graw Hill. 2003.

[3] Magos y Clarkson, Atomic absorption determination of total, inorganic and organic mercury in blood. J. Assoc. Offic. Anal. Chem. 55(1972) 966-971

[4] AOAC Official Method 977.15 Mercury in Fish. Alternative Flameless Atomic Absorption Spectrophotometric Method, First Action 1977, Final Action 1978.

[5] Sadiq, Sample weight and digestion 
temperature as critical factors in mercury determination in fish. Bull Environ. Contam. Toxicol. 47(1991) 335-341.

[6] Donna Megler, Contaminación en la Cuenca del rio nanay por efecto de la actividad minera. Institute des sciences de l'environment, Université du Québec à Montréal; http://www.uqam.ca/ (UQAM).

[7] Digesa, Información sobre niveles de metales pesados del rio Napo. Informe de ensayo $\mathrm{N}^{\circ}$ 3527 del 19-11-2003. Fecha de muestreo 15 11-2003, fecha de reporte 17-11-2003. e Informe $\mathrm{N}^{\circ} 3935-2010$

[8] Ávila P. P. Evaluación de los metales pesados $\mathrm{Cr}, \mathrm{Fe}, \mathrm{Ni}, \mathrm{Cu}, \mathrm{Zn}, \mathrm{Cd}, \mathrm{Pb}$ y $\mathrm{Hg}$ en agua, sedimento $y$ lirio acuático (Eichhorniacrassipes) de la Presa José Antonio Alzate, Estado de México. Tesis de
Maestría. Facultad de Química, Universidad Autónoma del Estado de México. (1995);102 p

[9] Chapman D. Water quality assessments. UNESCO, WHO, UNEP. New York, EUA. (1992); $585 \mathrm{p}$.

[10] Evaluación Mundial sobre el Mercurio, Programa de las Naciones Unidas para el medio ambiente. Publicado por el PNUMA Productos Químicos, Ginebra, Suiza, Diciembre. 2002;

[11] El Peruano - Norma legal establecida, Estándares Nacionales de Calidad Ambiental $\mathrm{N}^{\circ}$ 002-2008-MINAM.

E-mail: dumafarma@gmail.com 